\title{
Proteomic Analysis of Pelvic Autonomic Nerve in Nerve-sparing Radical Hysterectomy for Cervical Carcinoma
}

\author{
YOON HEE LEE ${ }^{1}$, MIN KYUNG KIM ${ }^{1}$, HEE YOUNG MOON ${ }^{1}$, GUN OH CHONG ${ }^{1}$, HYUN JUNG LEE ${ }^{1}$, \\ YOON SOON LEE ${ }^{1}$, JI YOUNG PARK ${ }^{2}$, CHAN HYEONG LEE ${ }^{3}$, MOON CHANG BAEK ${ }^{3}$ and DAE GY HONG ${ }^{1}$ \\ Departments of ${ }^{1}$ Obstetrics and Gynecology, and ${ }^{2}$ Pathology, School of Medicine, \\ Kyungpook National University, Kyungpook National University Chilgok Hospital, Daegu, Republic of Korea; \\ ${ }^{3}$ Department of Molecular Medicine, CMRI, School of Medicine, \\ Kyungpook National University, Daegu, Republic of Korea
}

\begin{abstract}
Background/Aim: This study was designed to identify candidate proteins which can be used for visualization of pelvic autonomic nerve during nerve-sparing surgery. Materials and Methods: Both soft tissue and vesical branch of the inferior hypogastric nerve from five women were collected during surgery. These 10 tissue specimens were analysed using liquid chromatography-mass spectrometry (LC-MS) and Human Protein Atlas (HPA) for protein expression. The existence of nerve fibres was confirmed using haematoxylin and eosin $(H \& E)$ and anti-S 100 staining. Results: A total of 413 proteins were detected. There were three proteins (isoform 1 of fibronectin, protein S100-A8 and A9) which implied a relation with pelvic autonomic nerve. In nerve tissue from one case, the existence of nerve fibre was not confirmed. Conclusion: Further large studies are expected to present more nerve-specific candidate proteins which can be used for the easy and safe identification of autonomic nerves.
\end{abstract}

Radical hysterectomy is the primary treatment for patients with locally advanced cervical carcinoma. Conventional radical hysterectomy has been considered to secure optimal radicality for prevention of recurrence. One of the most critical disadvantages of conventional radical hysterectomy

This article is freely accessible online.

Correspondence to: Dae Gy Hong, MD, Ph.D., Department of Obstetrics and Gynecology, School of Medicine, Kyungpook National University, Kyungpook National University Chilgok Hospital, Daegu, Republic of Korea. Tel: +82 532002681, Fax: +82 53200202, e-mail: dghong@knu.ac.kr

Key Words: Cervical carcinoma, nerve-sparing surgery, proteomics, liquid chromatography-mass spectrometry. is postoperative urinary dysfunction due to intraoperative injury of the pelvic autonomic nerves responsible for the neurological control of urinary function. Kobayashi in the 1960s first proposed the concept of autonomic nerve-sparing radical hysterectomy (NSRH) (1).

The efficacy and safety of NSRH are still controversial. There have been debates on the part of the uterine-supporting ligaments that the technique should be directed toward and concerns about whether NSRH may interfere with radicality (2). In addition, there is a discrepancy in effects of nervesparing between NSRH cases, even though NSRH may reduce the incidence of urinary dysfunctions. Incidences are different between studies (3).

The differences in the effect of NSRH are thought to be due to various factors including surgical technique. Surgeons sometimes cannot clearly distinguish pelvic autonomic nerves during surgery or fail to perform sufficient nervesparing surgery owing to excessive manipulation or cauterization (4).

It is not difficult to identify and isolate the inferior hypogastric nerve with the development of the surgical techniques and equipment. The most time-consuming and difficult part of nerve-sparing surgery is the dissection of the vesical branch of inferior hypogastric nerve. The vesical branch is vulnerable to injury during dissection and cauterization and is challenging to visualize. For this reason, preservation of the vesical branch is an essential part of nerve-sparing surgery. However, to our knowledge, there is no effective method to confirm sufficient preservation of the vesical branch during nerve-sparing surgery.

The purpose of this study was to identify the major proteins of the vesical branch of the inferior hypogastric nerve. It was designed as a basic study using proteomics to identify candidate proteins which can subsequently be used for visualization of pelvic autonomic nerve. 


\section{Materials and Methods}

Tissue samples, namely soft tissue and tissues from the vesical branch of the inferior hypogastric nerve, were collected from five women who underwent radical hysterectomy for locally advanced cervical carcinoma (Table I). Our operative technique and concept of NSRH have been described in detail elsewhere (5). The study was approved by the Institutional Review Board (approval number: KNUMC 14-1064) and performed with informed consents.

The surgeon biopsied a small portion of the tissue regarded as the vesical branch of the inferior hypogastric nerve using microendoscopic scissors after dissecting the vesical branch (Figure 1). The surgeon also biopsied soft tissues in the lateral pelvic wall. Two different tissue samples were subsequently stained with haematoxylin and eosin (H\&E) and for S100. The rest of the specimen was analysed using liquid chromatography-mass spectrometry (LC-MS).

Sample collection. Pelvic autonomic nerve and soft-tissue samples were collected during radical hysterectomy. The samples were collected into phosphate-buffered saline solution $\left[\mathrm{Na}_{2} \mathrm{HPO}_{4} \cdot 2 \mathrm{H}_{2} \mathrm{O}(3 \mathrm{mM}), \mathrm{NaCl}\right.$ (404 mM); $\mathrm{KH}_{2} \mathrm{PO}_{4}(1 \mathrm{mM})$, sodium azide $\left.(0.001 \%)\right]$. A protease inhibitor cocktail (Roche, Basel, Switzerland) comprising phenylmethylsufonyl fluoride $(200 \mathrm{mM}), N$-( $N$-(L-3-trans-carboxyirane-2-carbonyl)-L-leucyl)-agmatine $(10 \mathrm{mg} / \mathrm{ml})$ and pepstatin $(1$ $\mathrm{mg} / \mathrm{ml}$ ) was added to arrest proteolytic enzyme activity during sample storage. Samples were stored at $-80^{\circ} \mathrm{C}$ prior to extraction and analysis.

LC-MS sample preparation. To extract proteins from each tissue, $100 \mu \mathrm{l}$ of RIPA buffer ( $25 \mathrm{mM}$ Tris-HCl pH 7.6, $150 \mathrm{mM} \mathrm{NaCl}, 1 \%$ NP-40, $1 \%$ sodium deoxycholate, $0.1 \%$ SDS) was added to each tissue which was then homogenized by plastic tip homogenizer. The tissue debris was pelleted by centrifugation at $1,200 \times g$ for $15 \mathrm{~min}$ at $4^{\circ} \mathrm{C}$. Gel-assisted digestion of extracted proteins was performed as described previously (6). The proteins were chemically denatured by adding $10 \mathrm{mM}$ dithiothreitol and incubating at $60^{\circ} \mathrm{C}$ for $20 \mathrm{~min}$. Next, they were alkylated by adding $50 \mathrm{mM}$ iodoacetamide at room temperature and incubating for $20 \mathrm{~min}$. The protein solution was mixed with acrylamide/bisacrylamide solution (30\% v/v, 29:1) containing $10 \%(\mathrm{w} / \mathrm{v})$ ammonium persulfate and tetramethylethylenediamine. The resulting gel was cut into small pieces and washed with $50 \mathrm{mM}$ triethylammonium bicarbonate (TEABC) containing $50 \%$ acetonitrile $(\mathrm{ACN})$. Proteolytic digestion was performed using trypsin (protein:trypsin=50:1 w/w) in $50 \mathrm{mM}$ and incubating overnight at $37^{\circ} \mathrm{C}$. The digested peptides were extracted from the gel using sequential extraction with $200 \mu \mathrm{l}$ of $25 \mathrm{mM}$ TEABC, 200 $\mu \mathrm{l}$ of $0.1 \%$ TFA in water, $200 \mu \mathrm{l}$ of $0.1 \%$ TFA in ACN, and $200 \mu \mathrm{l}$ of $100 \%$ ACN. The eluents were concentrated using a Speedvac and desalted using HLB cartridge (Waters, Milford, MA, USA).

LC-MS data acquisition. Extracted peptides were analyzed by nanoUPLC (Waters, Manchester, UK) and tandem MS using Q-Tof Premier (Waters). Peptides were injected into a $2 \mathrm{~cm} \times 180 \mu \mathrm{m}$ trap column and resolved on a $25 \mathrm{~cm} \times 75 \mu \mathrm{m}$ nanoACQUITY C18 column (Waters) using the LC system. Mobile phase A was water with $0.1 \%$ and mobile B phase was $0.1 \%$ FA in ACN. The peptides were resolved using a gradient of $3 \%$ to $45 \%$ mobile phase B over $160 \mathrm{~min}$ at a flow rate of $300 \mathrm{nl} / \mathrm{min}$.

LC-MS data extraction and compound identification. For protein identification, MS raw data were converted into peak lists using
Table I. Clinical and surgical data of patients with cervical carcinoma $(n=5)$.

\begin{tabular}{lc}
\hline & Value \\
\hline Mean age (range), years & $51.3(38.4-65.6)$ \\
Body mass index (range), $\mathrm{kg} / \mathrm{m}^{2}$ & $24.2(18.5-27.2)$ \\
Histology, $\mathrm{n}$ & \\
Squamous cell carcinoma & 4 \\
Adenocarcinoma & 1 \\
Stage, $\mathrm{n}$ & 3 \\
IA2 & 2 \\
IB1 & $260(230-307)$ \\
Mean operative time (range), min & $150(110-200)$ \\
Blood loss, mean (range), ml & $0.8(0.5-1.1)$ \\
Mean haemoglobin change (range), g/dl & $6.7(5-11)$ \\
Mean self-voiding duration (range), days & $8.2(6-12)$ \\
Mean hospital stay (range), days &
\end{tabular}

MASCOT Distiller version 2.1 (Matrix Science, London, UK). All MS/MS raw data were analysed using MASCOT version 2.2.1 (Matrix Science). Mascot was set up to search the International Protein Index (IPI) HUMAN database version 3.78 (86,392 entries) (7). The database search against Mascot was performed with a fragment ion mass tolerance of $0.5 \mathrm{Da}$ and a parent ion tolerance of $0.2 \mathrm{Da}$. Two missed cleavages were allowed for trypsin digestion. Carbamidomethylation of cysteine residues and oxidation of methionine residues were considered as variable modifications. Peptide identities were assigned when their Mascot ion scores corresponded to values of $p<0.05$.

The proteins obtained by LC-MS were analysed using http://www. Proteinatlas.org. We reviewed whether the proteins obtained were related to the nerve using previously reported data via http://www.Proteinatlas.org and other studies which reported the relation between nerve and proteins.

The tissue block was cut into $7-\mu \mathrm{m}$ sections. Sections were stained with H\&E. Additional sections were selected for immunohistochemical analysis. Rabbit anti-S-100 (DaKo, Santa Clara, CA, USA) was used to identify peripheral nerves (Figure 2) (8).

\section{Results}

LC-MS analysis showed 413 proteins in 10 specimens from both groups. The detailed protein data are listed in supplementary materials available at https://blog.naver.com/ dghong0220/221295725390.

Overall, 130 proteins were identified in nerve tissues, 144 in the soft tissues, and 139 proteins were found in both. Of the 139 proteins found in both, nine were detected in all 10 tissue samples.

Six proteins were detected in all five soft-tissue samples, not in nerve tissues; seven proteins in four soft tissues; and 12 proteins in three soft-tissue samples.

No protein was common to all five nerve tissues samples. Five proteins were detected in four nerve tissue samples but not in soft tissues, namely ubiquitin-40S ribosomal protein 

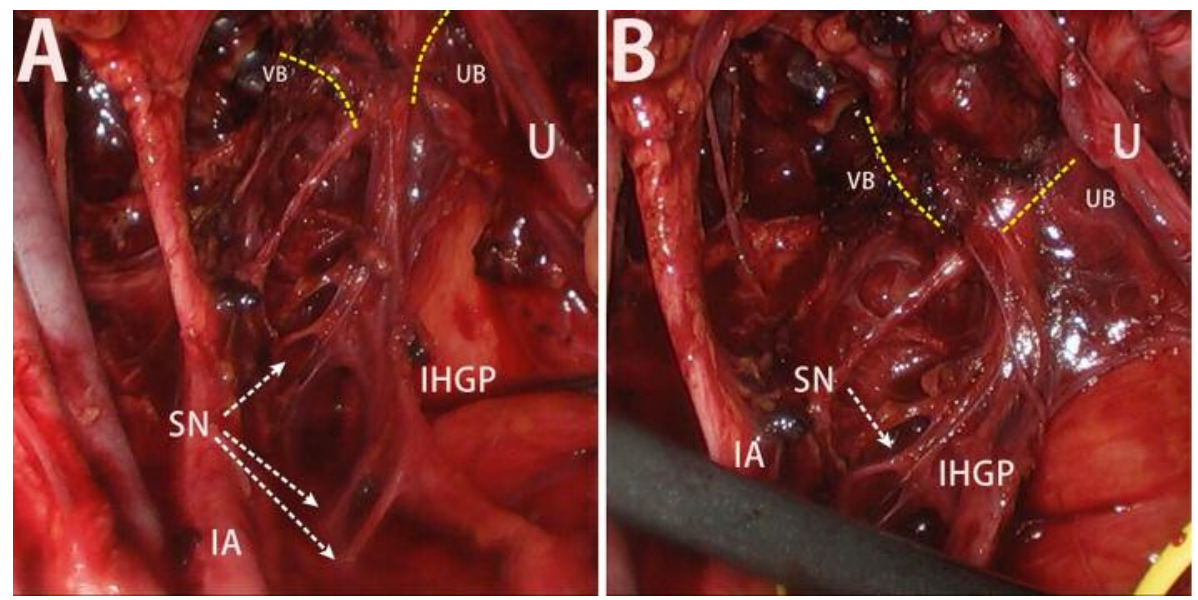

Figure 1. A: View of inferior hypogastric plexus, splanchnic nerves, vesical branch, and uterine branch of the inferior hypogastric nerve. B: Close view of vesical branch and uterine branch of the inferior hypogastric nerve. IA: Internal iliac artery, SN: splanchnic nerve, IHGP: inferior hypogastric plexus, VB: vesical branch, UB: uterine branch, U: ureter.
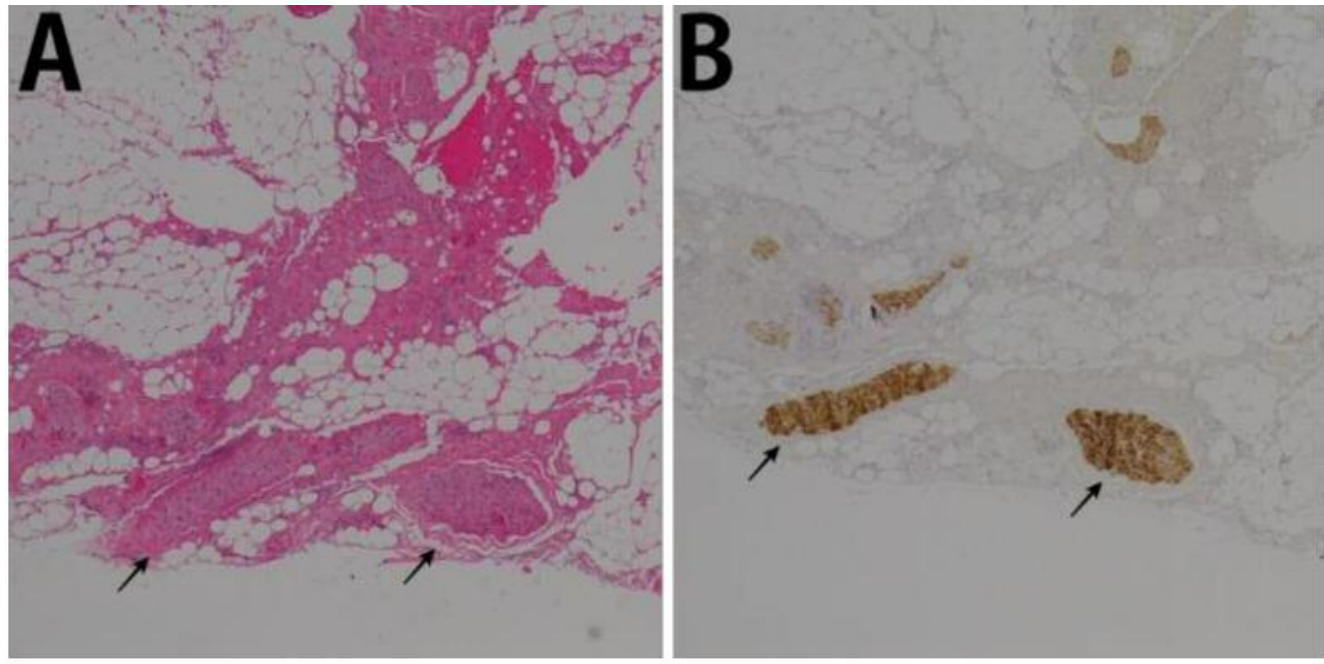

Figure 2. A: Haematoxylin and eosin stain for the vesical branch of the inferior hypogastric nerve. B: S100 protein immunohistochemical stain for the same portion of the vesical branch showing strong positivity (arrow). Original magnification, $\times 100$.

S27a, isoform alpha of caveolin-1, isoform 1 of myosin-9, protein S100-A9 and isoform 1 of gelsolin; and 11 proteins in three nerve tissues but not in soft tissues, namely calreticulin, isoform $\mathrm{H} 17$ of myeloperoxidase, cofilin-1, carbonyl reductase 3 , isoform 1 of fibronectin, protein S100-A8, alpha-1-acid glycoprotein 1, phosphatidylethanolamine-binding protein 1, haemoglobin subunit gamma-1, anti-(ED-B) scFV (fragment), LTF cDNA FLJ36533 fis and clone TRACH2004428, highly similar to lactotransferrin (fragment) (Table II) (Figure 3).

Nerve fibres were identified using H\&E and S100 staining in four nerve tissue cases, but nerve fibres were not identified in nerve tissue in one case. In soft tissue, nerve fibres were not identified with H\&E and S100 staining in any case. Among 16 proteins which were identified in three or more nerve tissues, five out of 16 proteins were expressed in peripheral nerve tissues via HPA analysis, namely ubiquitin-40S ribosomal protein S27a, carbonyl reductase 3, Isoform 1 of fibronectin, alpha-1-acid glycoprotein and isoform 1 of gelsolin.

\section{Discussion}

On the basis of anatomical knowledge and experience with cadavers, accurate nerve dissection and preservation is 
Table II. Summary of proteins detected in nerve tissue in at least three cases and staining for nerve tissue by case.

\begin{tabular}{|c|c|c|c|c|c|}
\hline Protein (protein score) & Case 1 & Case 2 & Case 3 & Case 4 & Case 5 \\
\hline Calreticulin & 62 & 51 & & & 51 \\
\hline Isoform $\mathrm{H} 17$ of myeloperoxidase & 56 & 62 & 47 & & \\
\hline Cofilin-1 & & & 60 & 75 & 37 \\
\hline Carbonyl reductase 3 & & 61 & & 87 & 51 \\
\hline Isoform 1 of fibronectin & 71 & 84 & 72 & & \\
\hline Protein S100-A8 & 77 & & 84 & & 84 \\
\hline Alpha-1-acid glycoprotein 1 & & & 89 & 90 & 75 \\
\hline Phosphatidylethanolamine-binding protein 1 & & 116 & 145 & 128 & \\
\hline Haemoglobin subunit gamma-1 & & 77 & 237 & & 88 \\
\hline Anti-(ED-B) scFV (fragment) & 243 & & & 116 & 90 \\
\hline cDNA FLJ36533 fis, clone TRACH2004428, & 145 & 73 & 299 & & \\
\hline Ubiquitin-40S ribosomal protein $\mathrm{S} 27 \mathrm{a}$ & & 47 & 62 & 45 & 37 \\
\hline Isoform alpha of caveolin-1 & & 52 & 54 & 70 & 39 \\
\hline Isoform 1 of myosin- 9 & 46 & 49 & 118 & 40 & \\
\hline Protein S100-A9 & 41 & 57 & 73 & & 198 \\
\hline Isoform 1 of gelsolin & 49 & & 175 & 117 & 73 \\
\hline \multicolumn{6}{|l|}{ Staining for nerve } \\
\hline $\mathrm{H} \& \mathrm{E}$ & + & + & + & - & + \\
\hline Anti-S100 & + & + & + & - & + \\
\hline
\end{tabular}

+ , Nerve fibres stained; -, nerve fibres not stained.

attempted during surgery. Surgeons have tried to ensure nerve preservation using a magnifying glass and following the pathway of nerves deep in the pelvis. Nerve-sparing surgery relies on surgical techniques used by surgeons. Its accuracy and objectivity are controversial. The most important part of nerve preservation during radical hysterectomy is preserving the vesical branch of the inferior hypogastric nerve.

The vesical branch of the inferior hypogastric nerve can be accessed after dissection of the posterior vesico-uterine ligament, which is anatomically rich in vascular structures and difficult to distinguish from the surrounding connective tissue.

Although researchers have reported their own methods for nerve preservation, standardized techniques and how to objectify nerve-sparing procedures during surgery have not yet been established.

Experimental studies have reported the objectification of nerve-preserving procedures. An anatomical and functional visualization of the structure of the inferior hypogastric nerve has been reported using immunohistochemistry with neuronal immuno-labelling, and computer-assisted 3D reconstruction (9). The report suggested that inferior hypogastric nerve has higher rate of coexistence of adrenergic and cholinergic nerve fibres toward the distal end. The anatomy of inferior hypogastric nerve was confirmed by analysing postoperative data. However, it was not helpful for practical nerve preservation during surgery.

Another method for confirming the nerve preservation during or after surgery is by evaluating the function of the organs during or after surgery using intraoperative electrical

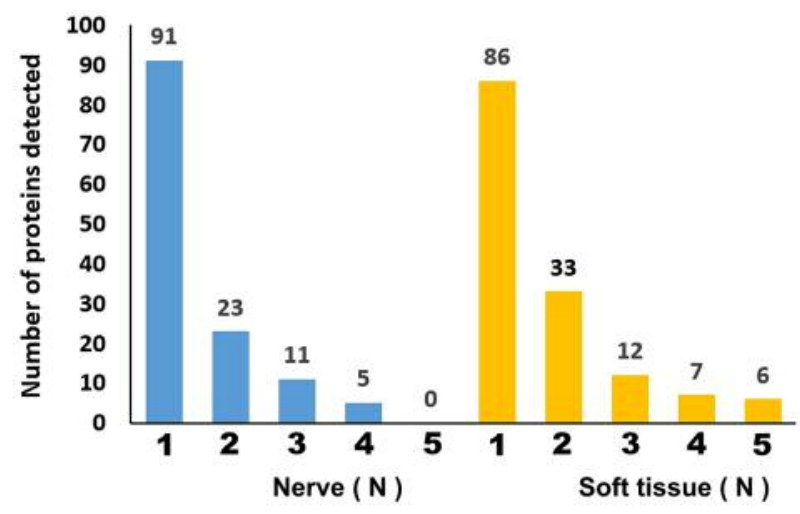

Figure 3. Number of proteins detected according to tissue sample type (nerve tissue and soft tissue from five cases). A total of 413 proteins were detected: 130 only in nerve tissue, 144 only in soft tissue, and 139 in both.

stimulation. Methods for identifying pelvic autonomic nerve preservation by measuring intraoperative vesical detrusor electromyographic activity have been considered useful (10, 11). However, intraoperative electrical stimulation is an indirect method for evaluating nerve preservation.

Efforts have been made to visualize nerves during surgery and directly identify nerve damage. In their study, Whitney et al. tried to visualize nerves during surgery in animal models. They used phage display to select a peptide that binded preferentially to nerves. After systemic injection of a 
fluorescently labelled version of the peptide in mice, all peripheral nerves were delineated. No changes in behaviour or activity were observed after treatment, indicating a lack of obvious toxicity (12). The fluorescent probe also labels nerves in human tissue samples. Fluorescence highlighting is independent of axonal integrity, suggesting that the probe could facilitate surgical repair of injured nerves and help prevent accidental transection.

However, to our knowledge, no reports have been published on fluorescent biomarkers for in vivo study.

In the present study, of the five proteins identified in nerve tissue from four cases, ubiquitin-40S ribosomal protein S27a and isoform 1 of gelsolin were expressed in peripheral nerves when retrieved via http://www.Proteinatlas.org. Caveolin-1 and myosin-9 were not expressed in peripheral nerves. S100 proteins are expressed exclusively in vertebrates, and exhibit somewhat cell-specific distribution (13). There are at least 21 different S100 proteins (14-17).

According to http://www.Proteinatlas.org, S100-A9 protein is not expressed in peripheral nerves. However, we found S100-A9 to be expressed in nerve tissue from four cases, suggesting a relationship with nerve tissue. Protein S100-A8/9 complex is an important initiator of immune responses in injured peripheral nerve Schwann cells. Protein S100-A8/9 complex leads to the following process. Immediately after injury, the chemokine-cytokine network is up-regulated in Schwann cells, and an initial chemotactic gradient is formed so that haematogenous immune cells are attracted to the site of injury (18).

In the present study, S100-A8 was expressed only in nerve tissue in three cases, and S100-A9 was expressed simultaneously with S100-A8. Proteins S100-A8 and A9 are presumed to be related to the pelvic autonomic nerve according to $\mathrm{H} \& \mathrm{E}$ and $\mathrm{S} 100$ stain for nerve fibres, as well as the review of HPA and literature (18). To our knowledge, immunohistochemical expression in the peripheral nerve has not been reported. Therefore, we suggest the need for further study on the relation between S100-A8/A9 and peripheral nerve tissue.

Three of the proteins expressed in nerve tissue of three cases have been reported to be expressed in nerve tissue by http://www.Proteinatlas.org. Among the three proteins, only carbonyl reductase 3 was expressed at a medium intensity at http://www.Proteinatlas.org. The other two proteins (isoform 1 of fibronectin, alpha-1-acid glycoprotein 1) showed a low intensity of expression. Although Isoform 1 of fibronectin is expressed in a low intensity at http://www.Proteinatlas.org. it seems to be related to pelvic autonomic nerves. Its expression pattern implied a relationship with the nerve. Nerve tissue in three cases cases which expressed Isoform 1 of fibronectin showed nerve fibres stained by $\mathrm{H} \& \mathrm{E}$ and for S100, and expressed S100-A8 and A9. Isoform 1 of fibronectin was not expressed in the case where the existence of nerve was not confirmed (Table II). The other proteins (ubiquitin-40S ribosomal protein $\mathrm{S} 27 \mathrm{a}$, carbonyl reductase 3, alpha-1-acid glycoprotein 1 and Isoform 1 of gelsolin) need further studies to infer their relationship with pelvic autonomic nerves.

In the tissue sampled as nerve tissue for one case, $\mathrm{H} \& \mathrm{E}$ and S100 staining showed no nerve tissue in the sample. These results mean that the surgeon's visual and empirical identification of pelvic autonomic nerve can be inaccurate.

We acknowledge that there are limitations to this study. Firstly, we only used previously reported nerve-related proteins and staining methods, which resulted in the limited identification of proteins related to peripheral nerve tissue. In addition, we obtained and used minimal tissue collection during surgery to minimize injury to the nerve fibres. Although during collection the tissue was presumed to be nerve tissue, the expressed proteins in tissues regarded as nerves should be analysed bearing in mind that unrelated proteins might be admixed because of the inaccuracies of tissue harvesting and the mixing of other tissue components.

Our results are insufficient to state powerful candidate proteins for visualization of pelvic autonomic nerves. However, this study is meaningful as an initial study to distinguish between nerve and surrounding tissues using proteins related to nerve tissue. Further large studies are expected to present more specific proteins related to nerve tissue

\section{Acknowledgements}

This study was funded by supported by a Biomedical Research Institute grant from Kyungpook National University Hospital (2014). The Authors declare that there are no conflicts of interest in this study. All procedures performed in studies involving human participants were in accordance with the ethical standards of the institutional and/or national research committee and with the 1964 Helsinki declaration and its later amendments or comparable ethical standards. All participants provided their informed consents for this study.

The authors have no commercial, proprietary, or financial interests in the products or companies described in this article. This work was supported by Biomedical Research Institute grant Kyungpook National University Hospital (2014).

\section{References}

1 Kobayashi T: Abdominal radical hysterectomy with pelvic lymphadenectomy for cancer of the cervix. Nanzando, Tokyo, pp. 178-187, 1961.

2 Kato T, Murakami G and Yabuki Y: A new perspective on nervesparing radical hysterectomy: Nerve topography and overpreservation of the cardinal ligament. Jpn J Clin Oncol 33(11): 589-591, 2003.

3 Kyo S, Kato T and Nakayama K: Current concepts and practical techniques of nerve-sparing laparoscopic radical hysterectomy. Eur J Obstet Gynecol Reprod Biol 207: 80-88, 2016. 
4 Kim HS, Kim K, Ryoo SB, Seo JH, Kim SY, Park JW, Kim MA, Hong KS, Jeong CW, Song YS and Group FS: Conventional versus nerve-sparing radical surgery for cervical cancer: A metaanalysis. J Gynecol Oncol 26(2): 100-110, 2015.

5 Park NY, Cho YL, Park IS and Lee YS: Laparoscopic pelvic anatomy of nerve-sparing radical hysterectomy. Clin Anat 23(2): 186-191, 2010.

6 Song MN, Moon PG, Lee JE, Na M, Kang W, Chae YS, Park JY, Park H and Baek MC: Proteomic analysis of breast cancer tissues to identify biomarker candidates by gel-assisted digestion and label-free quantification methods using LC-MS/MS. Arch Pharm Res 35(10): 1839-1847, 2012.

7 Kersey PJ, Duarte J, Williams A, Karavidopoulou Y, Birney E and Apweiler R: The International Protein Index: an integrated database for proteomics experiments. Proteomics 4(7): 19851988, 2004.

8 Schiffer D, Giordana MT, Mauro A and Migheli A: Immunohistochemistry in neuro-oncology. Basic Appl Histochem 30(2): 253-265, 1986.

9 Alsaid B, Bessede T, Karam I, Abd-Alsamad I, Uhl JF, Benoit G, Droupy S and Delmas V: Coexistence of adrenergic and cholinergic nerves in the inferior hypogastric plexus: Anatomical and immunohistochemical study with $3 \mathrm{D}$ reconstruction in human male fetus. J Anat 214(5): 645-654, 2009.

10 Chen CL, Guo HX, Yu YH, Liu P, Huang JS, Lu L, Guo Y, Liu W, Qi ST, Pan SY and Hou GN: The measurement of vesical detrusor electromyographic activity during nerve-sparing radical hysterectomy. Reprod Sci 17(12): 1144-1152, 2010.

11 Katahira A, Niikura H, Kaiho Y, Nakagawa H, Kurokawa K, Arai Y and Yaegashi N: Intraoperative electrical stimulation of the pelvic splanchnic nerves during nerve-sparing radical hysterectomy. Gynecol Oncol 98(3): 462-466, 2005.
12 Whitney MA, Crisp JL, Nguyen LT, Friedman B, Gross LA, Steinbach P, Tsien RY and Nguyen QT: Fluorescent peptides highlight peripheral nerves during surgery in mice. Nat Biotechnol 29(4): 352-356, 2011.

13 Donato R: S100: A multigenic family of calcium-modulated proteins of the EF-hand type with intracellular and extracellular functional roles. Int J Biochem Cell Biol 33(7): 637-668, 2001.

14 Wilson AJ, Maddox PH and Jenkins D: Cd1a and s100 antigen expression in skin langerhans cells in patients with breast cancer. J Pathol 163(1): 25-30, 1991.

15 Coppola D, Fu L, Nicosia SV, Kounelis S and Jones M: Prognostic significance of p53, BCL-2, vimentin, and S100 protein-positive Langerhans cells in endometrial carcinoma. Hum Pathol 29(5): 455-462, 1998.

16 Shinzato M, Shamoto M, Hosokawa S, Kaneko C, Osada A, Shimizu M and Yoshida A: Differentiation of Langerhans cells from interdigitating cells using CD1A and S-100 protein antibodies. Biotech Histochem 70(3): 114-118, 1995.

17 Marenholz I, Heizmann CW and Fritz G: S100 proteins in mouse and man: From evolution to function and pathology (including an update of the nomenclature). Biochem Biophys Res Commun 322(4): 1111-1122, 2004.

18 Chernov AV, Dolkas J, Hoang K, Angert M, Srikrishna G, Vogl T, Baranovskaya S, Strongin AY and Shubayev VI: The calciumbinding proteins S100A8 and S100A9 initiate the early inflammatory program in injured peripheral nerves. J Biol Chem 290(18): 11771-11784, 2015

Received May 13, 2018

Revised June 12, 2018

Accepted June 14, 2018 